\title{
The dynamics of magnetic resonance findings in patients with a spinal haematoma
}

\author{
R Firsching MD LRCP MRCS, ${ }^{1}$ F Zanella MD,${ }^{2} \mathrm{H}$ Lanfermann $\mathrm{MD}^{2}$ \\ ${ }^{I}$ Neurochirurgische Klinik, Ruhruniversität, In der Schornau 23-25, 4630 Bochum 7, \\ Germany; ${ }^{2}$ Radiologisches Institut der Universität Cologne, Germany.
}

In 6 patients with an incomplete transverse section, magnetic resonance (MR) imaging revealed a spinal haematoma. The MR signal intensity changed with the interval between the haemorrhage and the MR investigation. Signal intensities of T1- and T2-weighted images exhibited specific changes with time after the haemorrhage, allowing for a retrospective assessment of the time of onset of the haemorrhage.

Keywords: spinal haematoma; magnetic resonance imaging; signal intensity

\section{Introduction}

As spinal haematomas are infrequent, little experience with magnetic resonance (MR) imaging is available. The purpose of this report is to give an account of the MR findings in 6 patients with spinal haematomas.

\section{Patients}

\section{Case 1}

A 75 year old male patient was on dicumarol medication after a myocardial infarction and he suddenly developed pain in his neck and transient weakness of his legs. Two days later a sudden complete transverse lesion from segment C5 was noted, and the anticoagulation was discontinued. T1-weighted MRI on the third day revealed a space occupying lesion of low signal intesity dorsal to the cord extending from segment C5 to T2 (Fig 1). Immediately thereafter an extradural haematoma dorsal to the dural sac was removed.

\section{Case 2}

A 65 year old male patient with a history of transient ischaemic attacks woke up one night with sudden pain in the lower back. On admission a complete sensory and motor lesion at segment L1 was found. T2-weighted MR revealed a space occupying lesion of low, in-

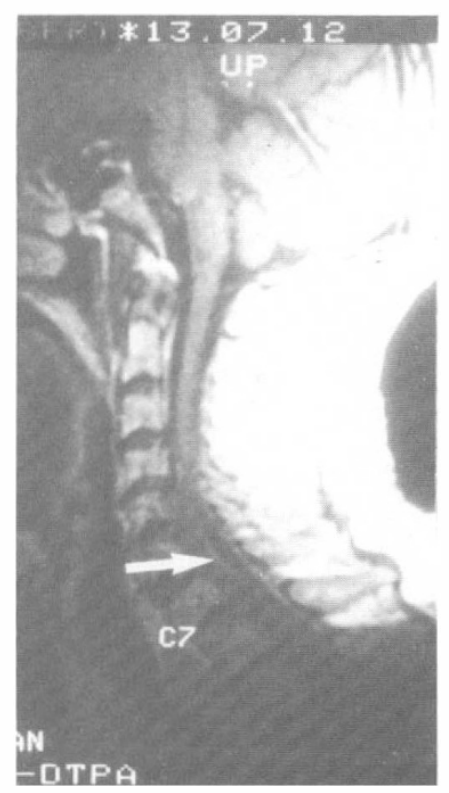

Figure 1 T1-weighted image revealing space occupying lesion from segment C5 to T2 presumably on the third day after a spinal haemorrhage.

homogenous signal intensity extending from T10 to L1 on the second day after the onset of paralysis. At operation an extradural haematoma dorsal to the dural sac was encountered. 


\section{Case 3}

The source of intermittent pain and weakness of both legs with free intervals could not be identified in this 57 year old male patient for 4 years. Fifteen days prior to admission he suddenly developed a complete transverse lesion at T6 level. On admission, MR demonstrated a space occupying lesion from segment C6 to T2. On T1-weighted images the signal intensity was low but inhomogenous, on T2-weighted images the signal intensity was mostly low (Fig 2a, b). Immediate laminectomy revealed an intramedullary liquid haematoma enclosed within a thin membrane. The cause of the haemorrhage was not found.

\section{Case 4}

This 24 year old male patient experienced two severe attacks of thoracolumbar region pain within 2 weeks. On the second occasion, he developed an incomplete transverse lesion syndrome, which resolved within 4 days. Lumbar myelography up to segment L1 failed to reveal any pathological abnormality. MR, however, demonstrated on T2-weighted images a space occupying lesion dorsal to the dural sac at L1 level. T1-weighted images displayed a low signal intensity lesion with a high signal intensity circumferential rim; while T2-weighted images only indicated an area of low signal intensity (Fig 3a,b). Aside from some pain there were no neurological deficits. Laminectomy revealed an organised haematoma, which was confirmed by histology. An arteriovenous malformation could not be verified. The origin of the haematoma remained unclear.

\section{Case 5}

This 64 year old male patient developed sudden weakness of all extremities and neck pain. He did not consult a physician until 13 days later, when MR disclosed a haematoma extending from C3 to T6 (Fig 4a-c) with a high signal intensity with $\mathrm{T} 1$ - and T2-weighted images. Operative removal was rejected.

\section{Case 6}

A lumbar puncture was performed in this 20 year old male patient who had acute lymphatic leukaemia, to obtain cerebrospinal fluid for further investigations. The next day increasing pain and weakness of both legs was noted. On

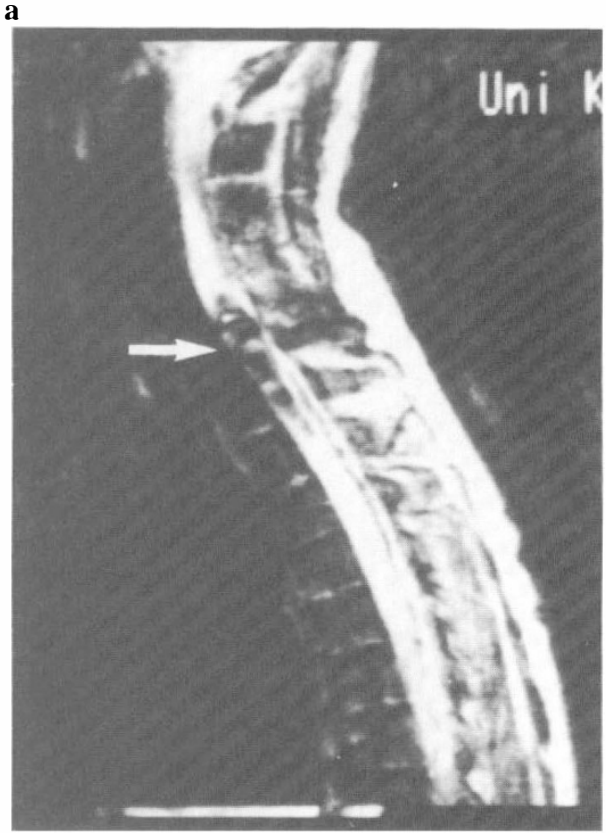

b

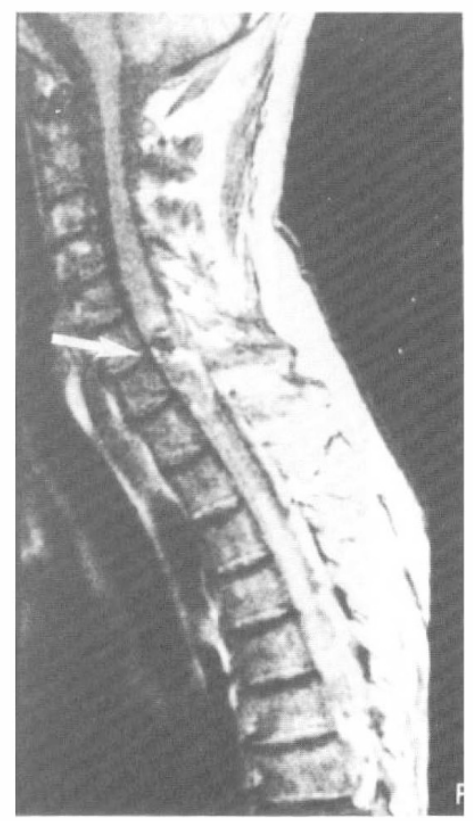

Figure 2 (a) T2-weighted image revealing an inhomogenous, mostly low signal intensity space occupying lesion extending from segment C6 to T2 on the second day after the onset of paralysis. (b) The same inhomogenous lesion on T1-weighted image. 
a

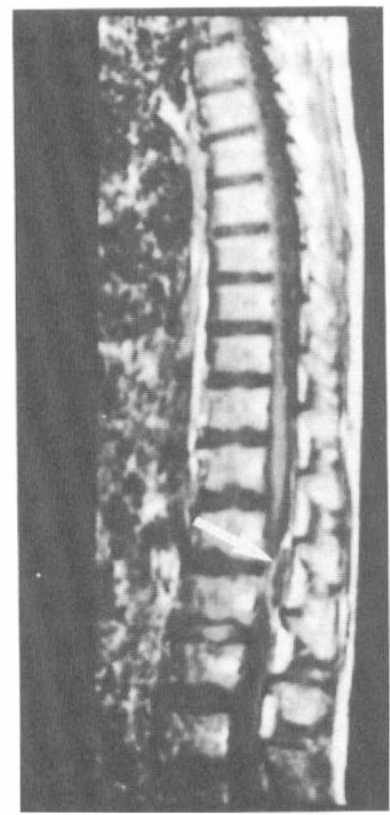

b

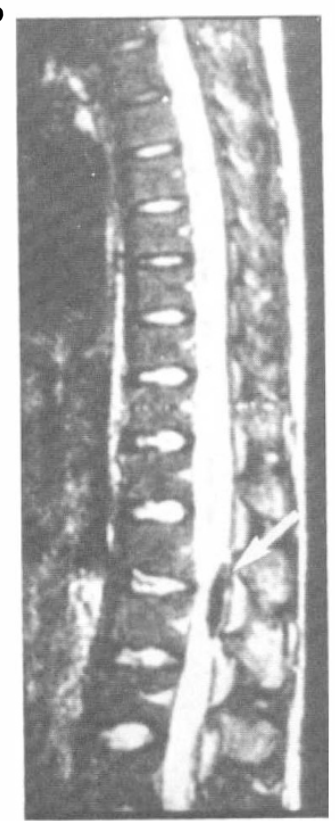

Figure 3 (a) T1-weighted image revealing an extramedullary epidural mass reaching from L1 to L2 probably caused by an acute haemorrhage 2 weeks and 4 days earlier. The inhomogenous lesion indents the dural sac, displacing the conus and cauda equina. The low signal intensity centre is enclosed in a high signal intensity rim on T1-weighted images. (b) Low signal intensity on T2-weighted images. the eleventh day after the lumbar puncture MR revealed an inhomogenous lesion extending from the conus to L5 with high signal intensities on T1- and T2-weighted images (Fig 5). Operative removal was rejected. A synopsis of the MR findings as related to the interval between the haemorrhage and the time of investigation is presented in Table I.

\section{Discussion}

While peridural and subdural intraspinal haematomas are not frequent, they are not particularly rare $^{1-7}$ From the literature available to us, there are only reports on MR in patients with an intramedullary haemorrhage, ${ }^{8.9}$ and none on those with an extramedullary spinal haematoma.

The MR signal intensity of any haematoma changes along with its degradation until it is resolved regardless of the cause and the location of the haemorrhage. This change of signal intensity is not yet fully understood, but appears to follow a regular pattern. ${ }^{10.11}$ In those with an acute haematoma up to 2 days after the haemorrhage, deoxyhaemoglobin $(\mathrm{Fe} 2+)$ is still restricted to the intact red blood cells. At this stage the paramagnetic effect must be weak because of the long distance between the iron and the hydrogen molecules with low signal intensities on T1-weighted images. Low signal intensity on $\mathrm{T} 2$-weighted images results from local changes within the magnetic field caused by variable tissue magnetisation in the same measurement volume due to the nonuniform distribution of iron between the intracellular and extracellular space. The resulting dephasing of the protons causes a shortening of $\mathrm{T} 2$ and decreasing signal intensity on $\mathrm{T} 2$-weighted scans. This $\mathrm{T} 2$ effect is more pronounced with higher magnetic field strengths. ${ }^{10.11}$ T1-weighted sequences are usually unable to identify acute haematomas, because this loss of signal is a $\mathrm{T} 2$ effect. The only T1-weighted finding, as in case 1, may be dislocation of the spinal cord, due to the space occupying effect of the haematoma.

In the subacute stage, 3-14 days after the haemorrhage, a rising signal intensity results from cell lysis and subsequent accumulation of methaemoglobin $(\mathrm{Fe} 3+)$, because 
a

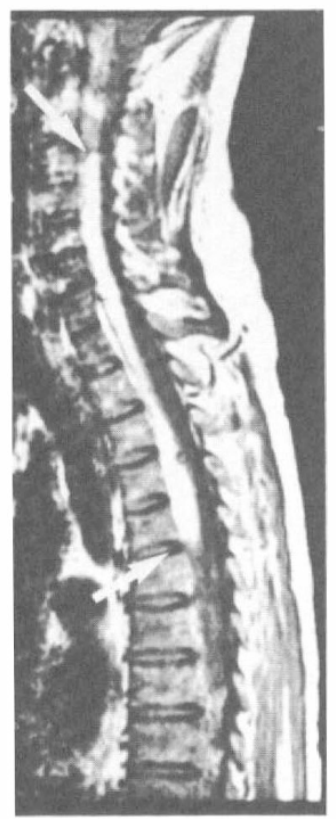

b

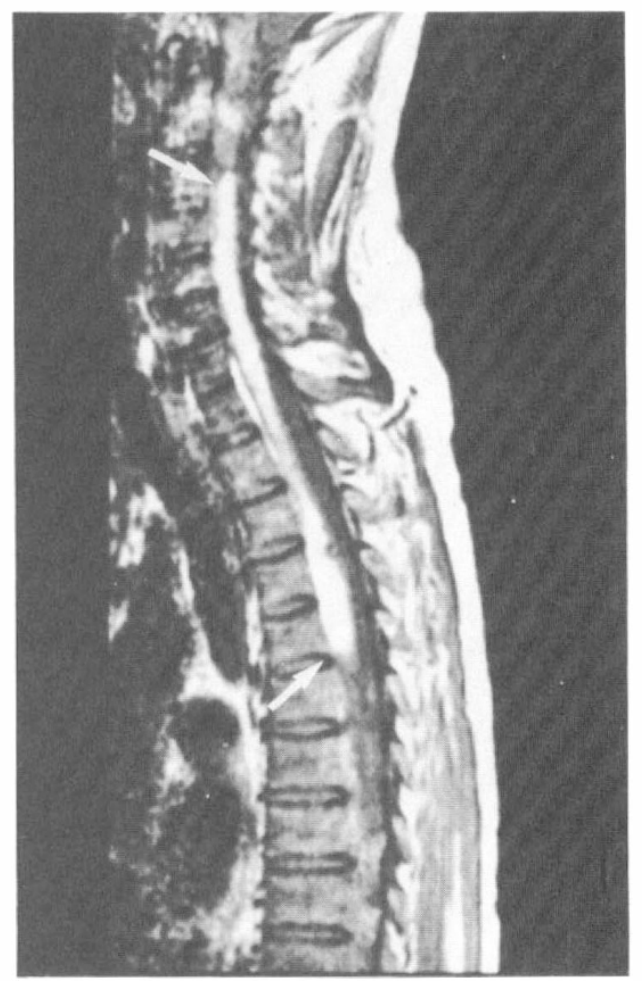

c

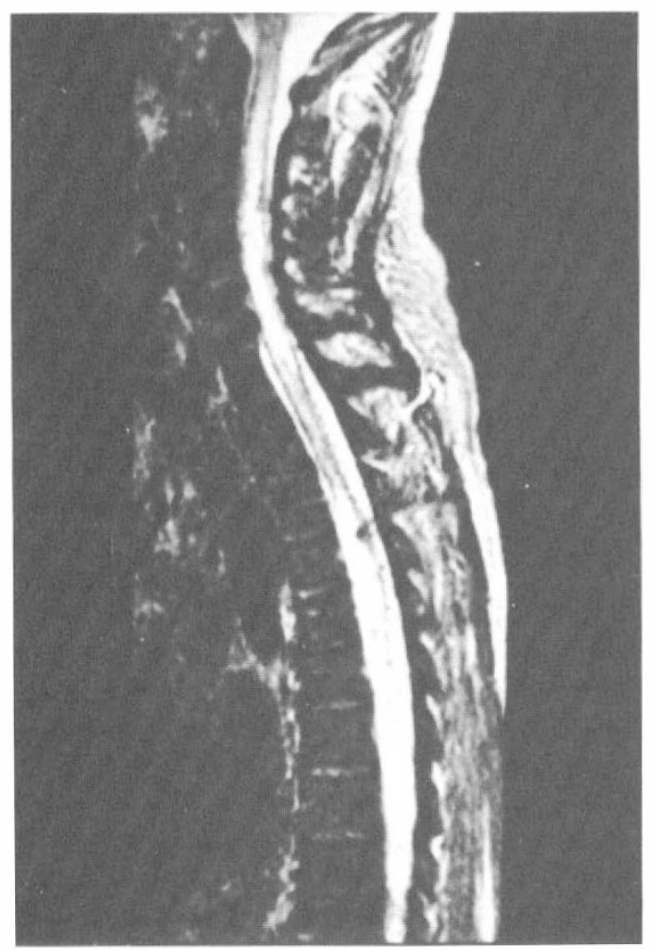

Figure 4 (a) Extensive high signal intensity lesion on T1-weighted images reaching from segment C3 to T6 13 days after a presumed haemorrhage. (b) Similar appearance on proton-weighted images. (c) High signal intensity also on T2-weighted images. 
$\mathbf{a}$

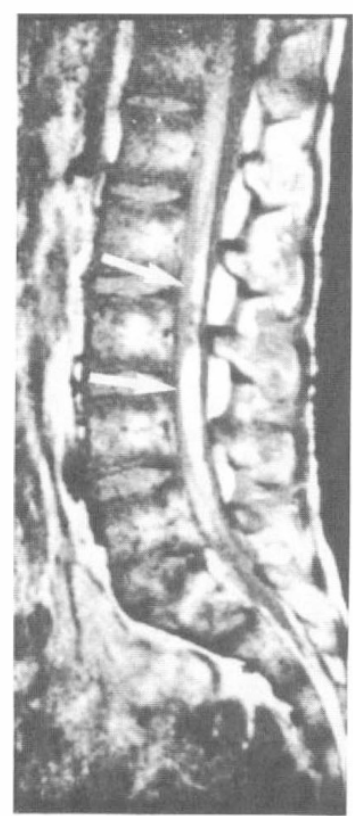

$\mathbf{b}$

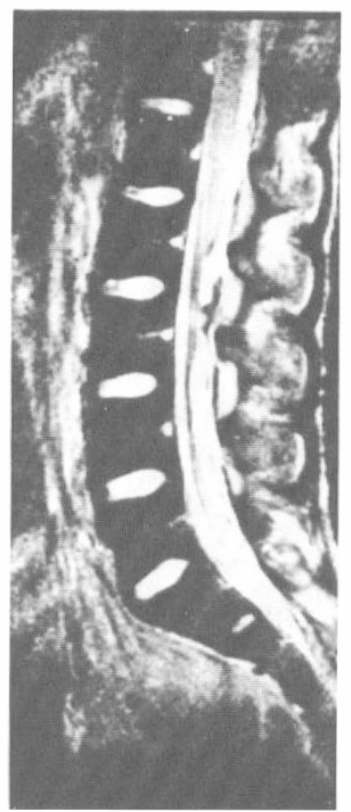

Figure 5 High signal intensity lesion extending from conus to L5 on (a) T1- and (b) T2-weighted images 11 days after presumed haemorrhage. In addition, inhomogenous signal intensities of vertebral bone marrow in acute leukaemia.

Table I

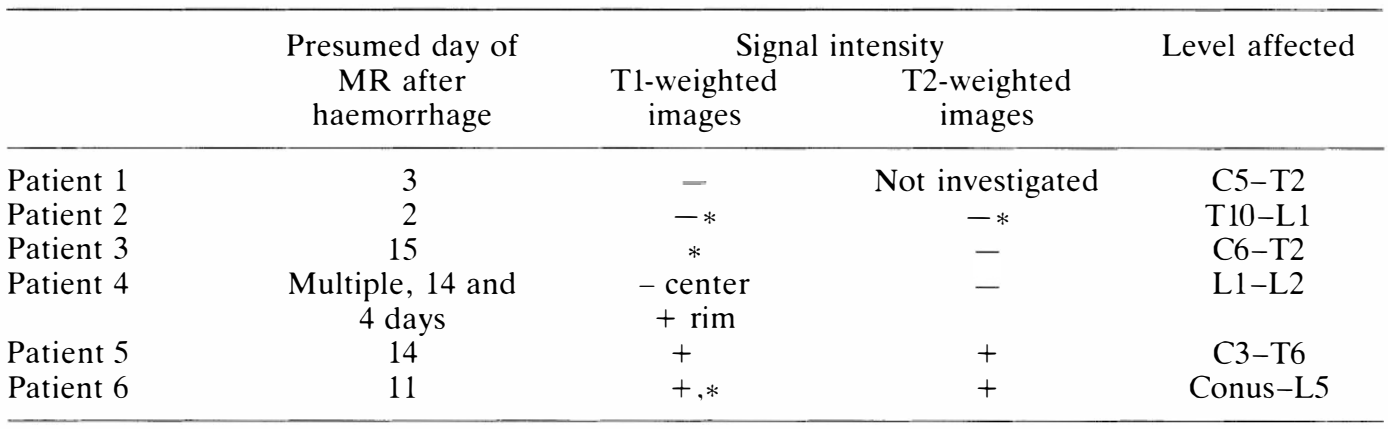

+ High signal intensity

- Low signal intensity

* Inhomogenous signal intensity

methaemoglobin cannot bind oxygen and has a strong paramagnetic effect.

After 6-8 days the haemorrhage is revealed by an intense signal on both T1- and T2-weighted images because of a further prolongation of the relaxation times. The rise of signal intensity becomes particularly apparent in short TR/TE sequences, because the covalent binding of an $\mathrm{OH}$ group tends mainly to shorten the $\mathrm{T} 1$ relaxation time.

At the end of the subacute stage, haemosiderin deposits may cause a signal loss at the periphery of the zone of high signal 
intensity. This phenomenon is explained by inhomogeneities of tissue magnetisation.

\section{Conclusion}

MR findings in spinal haematomas are related to time. They depend on the interval between the acute haemorrhage and the MR investigation. In the first days a loss of signal intensity is prominent, in the subacute stage signal intensity increases on T1- and $\mathrm{T} 2$-weighted images and decreases in the later stages.

\section{References}

1 Edelson RN (1976) Spinal subdural hematoma. In: Vinken P, Bruyn G, editors. Handbook of Clinical Neurology. Vol 26, Injuries of the Spine and Spinal Canal. North Holland Publishing Company, Amsterdam: 31-37.

2 Guthikonda M, Schmidek H. Wallman L, Snyder T (1979) Spinal subdural hematoma: Case report and review of the literature. Neurosurg 5: 614-616.

3 Harik S, Raichle M, Reis D (1971) Spontaneous remitting spinal epidural hematoma in a patient on anticoagulants. $N$ Eng J Med 284: 1355-1357.

4 Paredes E, Kishore P, Ward J (1981) Cervical spinal subdural hematoma. Surg Neurol 15: 477-479.

5 Piotrowski W, Kröger M, Tornow (1979) Das spinale epidurale Hämatom. Nervenarzt 50: 426-431.

6 Schiller F, Neligan G, Budtz-Olsen O (1948) Surgery in haemophilia: A case of spinal subdural haematoma producing paraplegia. Lancet 2: 842-845.

7 Tomarken JL (1987) Spinal subdural hematoma. Am J Emerg Med 5: 123-125.

8 Biller J, Gentra LR, Adams HP, Morris DC (1986) Spontaneous hemorrhage in the medulla oblongata: clinical MR correlations. J Comput Assist Tomogr 10: 303-306.

9 Naseem M, Zachariah SB, Stone J, Russell E (1986) Cervico-medullary hematoma: diagnosis by MR. AJNR 7: 1096-1098.

10 Hayman LA, Pagani JJ, Kirkpatrick JB, Hinck VC (1989) Pathophysiology of acute intracerebral and subarachnoid hemorrhage: Applications of MR imaging. Amer J Roentgenol 153: 135-139.

11 Seidenwurm D, Meng TK, Kowalski H, Weinreb JC. Kricheff II (1989) Intracranial hemorrhagic lesions: Evaluation with spin-echo and gradient-refocused MR imaging at 0.5 and 1.5 T. Radiology 172: 189-194. 